\title{
Ocorrência de animais com anticorpos anti-Leptospira spp. na região de Uberlândia, MG, 2011-2014
}

\author{
Alex Ferreira Mendes ${ }^{2}$, Ana Beatriz Garcez Buiatte ${ }^{3}$, Dayane Olímpia Gomes ${ }^{4}$, Andreia Zago \\ Ciuffa $^{5}$, Thais Fernanda Martins dos Reis ${ }^{6}$, Anna Monteiro Correia Lima ${ }^{7}$
}

Resumo: A Leptospirose é uma doença cosmopolita de grande relevância para a Medicina Veterinária por ocorrer em diversas espécies domesticas e silvestres, podendo causar prejuízos nos setores produtivos e preocupações para a saúde pública. Assim, o objetivo do trabalho foi avaliar a frequência de bovinos, bubalinos, caninos selvagens, caninos, caprinos, equinos, felinos, ovinos, roedores e suínos sororeagentes ao teste para leptospirose e quais sorogrupos foram mais prevalentes. Para tal, foram compilados os resultados dos exames de leptospirose obtidos pelo teste de Soroaglutinação Microscópica realizados no Laboratório de Doenças Infectocontagiosas da Faculdade de Medicina Veterinária da Universidade Federal de Uberlândia, entre os anos de 2011 a 2014. A espécie que apresentou maiores índices de soropositividade foi a bovina, o sorogrupo mais prevalente foi o Sejroe.

Palavras-chave: espécies; leptospirose; Teste de Soroaglutinação Microscópica.

\section{Occurrence of animals with anti-Leptospira spp. antibodies in the region of Uberlândia, MG, 2011-2014}

\begin{abstract}
Leptospirosis is a cosmopolitan disease, with a great importance to Veterinary Medicine by occurring in several domestic and wild species, it also may cause losses in the productive sectors and public health concerns. The objective of the study was to evaluate the frequency of cattle, buffaloes, wild canines, canines, goats, horses, cats, sheep, rodents and pigs reactive serum to test for leptospirosis, which serogroups were most prevalent. The results of leptospirosis exams obtained by the Microscopic Agglutination Test made in Infectious Diseases Laboratory of the Faculty of Veterinary Medicine of the Federal University of Uberlândia were compiled between the years of 2011 to 2014. The specie that showed higher rates of seropositivity was bovine, the most prevalent serogruoup in the tests was Sejroe.
\end{abstract}

Keywords: species; leptospirosis; Microscopic Agglutination Test.

\footnotetext{
${ }^{1}$ Submetido em 12/06/2018 e a provado em 04/08/2018

${ }^{2}$ Médico Veterinário; Residente em Diagnóstico por Imagem, Universidade Estadual Paulista Julio de Mesquita Filho (UNESP), BotucatuSão Paulo, CEP: 18618-681; E-mail: alex.f.mendes@ @otmail.com

${ }^{3}$ Especialista em Medicina Veterinária Preventiva; Mestranda Universidade Federal de Uberlândia (UFU), Programa de Pós-Graduação em Ciências Veterinárias, Uberlândia-Minas Gerais, CEP: 38405-315; E-mail: anabgbuiatte@ hotmail.com

${ }^{4}$ Mestre em Ciências Veterinárias, Doutoranda, Universidade Federal de Uberlândia (UFU), Programa de Pós-Graduação em Ciências Veterinárias, Uberlândia-Minas Gerais, CEP: 38405-315; E-mail: dayanevet@yahoo.com.br

${ }^{5}$ Mestre em Ciências Veterinárias; Doutoranda, Universidade Federal de Uberlândia (UFU), Programa de Pós-Graduação em Ciências Veterinárias, Uberlândia-Minas Gerais, CEP: 38405-315; E-mail: andreiazago@ yahoo.com.br

${ }^{6}$ Especialista em Medicina Veterinária Preventiva; Mestranda em Ciências Veterinárias, Universidade Federal de Uberlândia (UFU), Uberlândia-Minas Gerais, CEP: 38405-315; E-mail: thais_koro@hotmail.com

${ }^{7}$ Doutora em Ciências Veterinárias, Professora Adjunta, Universidade Federal de Uberlândia (UFU), Faculdade de Medicina Veterinária,

Uberlândia-Minas Gerais, CEP: 38405-315; E-mail: annalimaufu@yahoo.com.br
} 


\section{Introdução}

A leptospirose é uma zoonose de distribuição mundial, causada por bactérias do gênero Leptospira spp., compreendendo 19 espécies, que pode afetar animais domésticos e selvagens, e também seres humanos (Souza et al., 2016).

Segundo Castro (2010), a infecção depende dos fatores ambientais que permitam a sobrevivência da bactéria fora do hospedeiro. A doença é descrita como tendo alta prevalência em países de clima tropical, fato justificado pelos fatores ambientais, principalmente, por grandes precipitações pluviais e tipo de solo (Acha e Szyfres, 2003).

A leptospirose pode afetar várias espécies e trazer prejuízos para vários seguimentos onde atua o médico veterinário. No âmbito da produção, por exemplo, ela é capaz de gerar baixos índices produtivos, afetando significativamente o setor agropecuário; e para a saúde pública, ela é uma importante zoonose, trazendo riscos à saúde humana e dos animais de companhia (Eckstein et al., 2017).

A infecção sistêmica aguda é caracterizada por febre, insuficiência renal ou hepática, e ainda por problemas relacionados ao sistema reprodutivo;já com a cronicidade, a principal preocupação é a característica de portador renal assintomático, onde o animal continua eliminando a bactéria por anos pela urina, contaminando o ambiente e sendo um veiculador da doença, além de ser capaz de fazer a manutenção da doença no meio (Levett, 2001).

A intensidade da leptospirose também depende da competência imune do animal. Quanto mais debilitado, mais aguda é a doença e mais a bactéria se multiplica em seu organismo. Isso explica uma grande variação de titulação sanguínea de anticorpos anti-Leptospira spp. do mesmo sorogrupo na mesma espécie e em animais circunvizinhos (Adler, 2015).

$O$ teste de Soroaglutinação Microscópica (SAM) é o teste indicado pela Organização Mundial de Saúde Animal (OIE), e, por meio dele é possível identificar se o animal testado tem títulos de anticorpos contra a Leptospira spp., quantificá-los em várias diluições e ainda identificar a reação contra cada sorogrupo específico (OIE, 2014).
Diante do exposto, o objetivo deste trabalho foi realizar um estudo retrospectivo sobre a ocorrência de animais que apresentaram anticorpos anti-Leptospira spp. na microrregião de Uberlândia - MG a partir de testes de Soroaglutinação Microscópica em microscópio de campo escuro realizados no Laboratório de Doenças Infectocontagiosas da Faculdade de Medicina Veterinária da Universidade Federal de Uberlândia, entre os anos de 2011 a 2014, para avaliar a frequência de animais de diferentes espécies sororeagentes a prova e quais sorogrupos foram mais prevalentes nelas.

\section{Materiais e Métodos}

A equipe que trabalha no Laboratório de Doenças Infectocontagiosas da Faculdade de Medicina Veterinária da Universidade Federal de Uberlândia é composta por residentes, mestrandos e doutorandos, que ao realizarem testes de Soroaglutinação Microscópica para leptospirose, catalogam os resultados em um livro. Estes testes são realizados a partir de amostras provenientes do hospital veterinário da universidade, outros hospitais veterinários da região, de médicos veterinários autônomos e de pesquisas. A partir dos dados registrados e contabilizados nesse livro foi feito um estudo retrospectivo avaliando os resultados encontrados nos exames referentes a 4 anos (2011 - 2014).

Foram testados, durante o período de 2011 a 2014, 2230 animais de 10 diferentes espécies, sendo elas: bovinos, bubalinos, caninos selvagens, caninos, caprinos, equinos, felinos, ovinos, roedores e suínos.

Os testes realizados no Laboratório de Doenças Infectocontagiosas FAMEV/ UFU para leptospirose são de Soroaglutinação Microcópica em Microscópio de Campo Escuro (modelo Carl Zeiss Microlmaging $\mathrm{GmbH}$ ), e tem base a seguinte coleção de antígenos de Leptospira spp. sorogrupo Autumnalis (sorovares Autumnalis e Bratislava), sorogrupo Autumnalis (sorovares Autumnalis e Bratislava), sorogrupo Australis (sorovares Australis e Butembo), sorogrupo Bataviae (sorovar Batavie), sorogrupo Canicola (sorovar Canicola), sorogrupo Cynopteri (sorovar Cynopteri), sorogrupo Djasiman (sorovar Djasiman), sorogrupo Grippotyphosa (sorovar Grippotyphosa), sorogrupo Sejroe 
(sorovares Hardjo e Wolffi), sorogrupo Hebdomadis (sorovar Hebdomadis), sorogrupo Icterohaemorragie (sorovares Copenhageni e Icterohaemorragie), sorogrupo Javanica (sorovar Javanica), sorogrupo Panama (sorovar Panama), sorogrupo Semaranga (sorovar Patoc), sorogrupo Pomona (sorovar Pomona), sorogrupo Pyrogenes (sorovar Pyrogenes), sorogrupo Tarassovi (sorovar Tarassovi), e sorogrupo Celledoni (sorovar Whitcomb) (Adler, 2015). A técnica usada segue (Brasil, 1995) e foi descrita por Santos (2007).

Foram considerados positivos reagentes animais com anticorpos contra algum sorogrupo de leptospira, aqueles exames que reagiram em diluição de 1:100, já que esses animais poderiam estar doentes ou apenas apresentarem titulação vacinal, e positivos certamente doentes aqueles animais cujos exames apresentaram titulação a partir de 1:200 para qualquer sorogrupo da coleção testada (Brasil, 2009).

As análises estatísticas foram feitas utilizando-se o software SPSS (IBM SPSS, 2013) adotando-se significância de 5\%.
A análise da relação de casos positivos para leptospirose de acordo com o teste de Soroaglutinação Microscópica e as espécies animais foi feita por meio do teste Qui-quadrado para independência e teste de Comparação de Proporções entre as espécies.

Ao estudar cada sorogrupo, verificou-se a relação entre animais positivos para leptospirose de acordo com o teste de Soroaglutinação Microscópica (apresentando classificação 0, 1 ou 2) com as espécies animais estudadas por meio do teste Qui-quadrado e teste de Comparação de Proporções entre as espécies.

\section{Resultados e Discussão}

Conforme a Tabela 1, 621 animais $(27,8 \%)$ foram positivos para algum sorogrupo da coleção de leptospiras testadas e 1609 (72,2\%) não foram reagentes.

A espécie que mais apresentou casos reagentes foi a bovina, com $504(22,6 \%)$ animais positivos do total analisado.

Tabela 1 Relação entre soropositividade de animais reagentes ao teste SAM realizado no Laboratório de Doenças Infectocontagiosas FAMEV/ UFU, entre os anos de 2011 e 2014, e as 10 espécies animais testadas no estudo

\begin{tabular}{|c|c|c|c|c|c|c|c|c|c|c|c|}
\hline \multirow{2}{*}{$\begin{array}{l}\text { Frequência } \\
\text { de animais }\end{array}$} & \multicolumn{10}{|c|}{ Espécie } & \multirow[t]{2}{*}{ Total } \\
\hline & Bovinos & Bubalinos & $\begin{array}{c}\text { Caninos } \\
\text { selvagens }\end{array}$ & Caninos & Caprinos & Equinos & Felinos & Ovinos & Roedores & Suínos & \\
\hline \multirow{2}{*}{$\begin{array}{c}\text { Não } \\
\text { reagentes }\end{array}$} & $982_{a}$ & $14_{b}$ & $24_{a, b}$ & $222_{c}$ & $105_{d}$ & $28 \mathrm{a}, \mathrm{b}$ & $35_{\mathrm{d}, \mathrm{e}}$ & $39_{\mathrm{d}, \mathrm{e}, \mathrm{f}}$ & $130_{c, f}$ & $30_{c, e, f}$ & 1609 \\
\hline & $44,00 \%$ & $0,60 \%$ & $1,10 \%$ & $10,00 \%$ & $4,70 \%$ & $1,30 \%$ & $1,60 \%$ & $1,70 \%$ & $5,80 \%$ & $1,30 \%$ & $72,20 \%$ \\
\hline \multirow{2}{*}{ Reagentes } & $504 a$ & $18 \mathrm{~b}$ & $17 \mathrm{a}, \mathrm{b}$ & $43 c$ & $1 \mathrm{~d}$ & $17_{\mathrm{a}, \mathrm{b}}$ & $0_{\mathrm{d}, \mathrm{e}}$ & $1 \mathrm{~d}, \mathrm{e}, \mathrm{f}$ & $17_{c, f}$ & $3 \mathrm{c}, \mathrm{e}, \mathrm{f}$ & 621 \\
\hline & $22,60 \%$ & $0,80 \%$ & $0,80 \%$ & $1,90 \%$ & $0,00 \%$ & $0,80 \%$ & $0,00 \%$ & $0,00 \%$ & $0,80 \%$ & $0,10 \%$ & $27,80 \%$ \\
\hline \multirow{2}{*}{ Total } & 1486 & 32 & 41 & 265 & 106 & 45 & 35 & 40 & 147 & 33 & \\
\hline & $66,60 \%$ & $1,40 \%$ & $1,80 \%$ & $11,90 \%$ & $4,80 \%$ & $2,00 \%$ & $1,60 \%$ & $1,80 \%$ & $6,60 \%$ & $1,50 \%$ & \\
\hline
\end{tabular}

Letras iguais mostram relação estatísticas entre as espécies e letras diferentes mostram relações estatísticas diferentes.

Os dados se diferem dos encontrados por Martins e Lilenbaum (2013), que analisaram dados retrospectivos de exames de leptospirose dos últimos vinte anos na cidade do Rio de Janeiro, RJ - Brasil. Eles encontraram uma maior soropositividade em cães $(73,3 \%)$, sorogrupo Icterohaemorragiae. Isso mostra que a região estudada pelos autores proporciona uma maior facilidade para contaminação entre cães que o desta pesquisa, e que eles estão fazendo a manutenção da bactéria. Como se espera que a maior parte dos caninos testados seja da área urbana, os dados levantam preocupações em relação a fatores ambientais que podem funcionar como fatores de risco para a disseminação da doença, como por exemplo, as enxurradas, um grande problema encontrado pelas grandes cidades como o Rio de Janeiro.

Ainda neste estudo de Martins e Lilenbaum (2013), os suínos $(66,1 \%)$ e ovinos $(47,4 \%)$ também apresentaram alta soropositividade, maior que a encontada em bovinos (46,9\%). Ainda assim, bovinos tiveram uma média maior que a deste estudo. Isso reforça a teoria de alta disseminação da doença na zona rural. $\mathrm{O}$ sorogrupo mais encontrado pelos pesquisadores foi Sejroe. 
Os sorogrupos que tiveram maior reatividade foram Sejroe, com sorovar Hardjo com 390 $(17,45 \%)$ animais positivos e o sorovar Wolffi com $205(9,2 \%)$ animais positivos, e o sorogrupo Hebdomadis com 171 (7,7\%) animais positivos, conforme visualizado na Tabela 2.

Tabela 2 Distribuição de sorovares em animais reativos à soroaglutinação microscópica entre os anos de 2011 e 2014, em Uberlândia, MG, nas espécies estudadas: bovinos, bubalinos, caninos, caninos selvagens, caprinos, equinos, felinos, ovinos, roedores e suínos

\begin{tabular}{llccc}
\hline \multirow{2}{*}{ Sorogrupos } & Sorovares & \multicolumn{2}{c}{ Titulações $(\mathbf{\%})$} & Total (\%) \\
Sejroe & Hardjo & $\mathbf{1 : 1 0 0}$ & $\mathbf{1 : 2 0 0}$ & 17,45 \\
Hebdomadis & Wolffi & 8,70 & 8,70 & 9,20 \\
Grippothyphosa & Hebdomadis & 5,60 & 3,60 & 7,70 \\
Canicola & Grippothyphosa & 5,10 & 2,60 & 3,80 \\
Icterohaemorrhagiae & Canicola & 2,10 & 1,70 & 3,80 \\
Pomona & Icterohaemorrhagiae & 2,40 & 1,40 & 0,90 \\
Semaranga & Copenhageni & 2,20 & 1,60 \\
Tarassovi & Pomona & 0,50 & 0,40 & 1,30 \\
Pyrogenes & Patoc & 1,20 & 0,40 & 1,20 \\
Autumnalis & Tarassovi & 0,90 & 0,40 & 1,10 \\
& Pyrogenes & 0,80 & 0,40 & 0,90 \\
Australis & Autumnalis & 0,70 & 0,40 & 0,05 \\
Bataviae & Butembo & 0,60 & 0,30 & 0,60 \\
Djasiman & Australis & 0,05 & 0 & 0,50 \\
Celledoni & Bratislava & 0,30 & 0,30 & 0,60 \\
Javanica & Bataviae & 0,40 & 0,10 & 0,25 \\
Panama & Djasiman & 0,40 & 0,20 & 0,20 \\
Cynopteri & Whitcomb & 0,20 & 0,05 & 0,15 \\
\hline & Javanica & 0,20 & 0 & 0,15 \\
& Panama & 0,10 & 0,05 & 0,05 \\
\hline
\end{tabular}

Em relação às espécies, 504 bovinos foram positivos e o sorogrupo mais reagente foi o Sejroe, com 347 animais reagentes ao sorovar Hardjo $(68,85 \%)$ e 191 animais reagentes ao sorovar Wolffi $(37,9 \%)$ e ainda o sorogrupo Hebdomadis, com $144 \quad(28,57 \%)$ animais positivos. Oliveira et al. (2010), ao avaliarem 1076 bovinos no estado da Bahia, o sorogrupo mais prevalente foi Sejroe, assim como foi visto neste estudo. Também em estudo de Souza et al. (2016), em Uberlândia, MG, o sorogrupo mais prevalente em bovinos foi Sejroe. A distribuição deste sorogrupo em bovinos, praticamente homogênea, parece ser independente das diferentes condições de cada região (Souza et al., 2016).

Dentre os 18 bubalinos positivos, os sorogrupos de maior prevalência também foram o sorogrupo Sejroe, com 15 (83,33\%) animais reagentes aos sorovar Hardjo e $10(55,56 \%)$ ao sorovar Wolffi, e o sorogrupo Hebdomadis, com seis $(33,33 \%)$ animais positivos. Resultados de pesquisas no estado de São Paulo demonstram sorogrupo Sejroe como o mais prevalente em bubalinos (Favero et al., 2002; Langoni et al., 1999). Oliveira et al. (2013) observaram maior reatividade para os sorogrupos Djasiman, Sejroe e Australis no estado do Pará. Segundo Bharti et al. (2003), as diferenças nos achados podem estar relacionadas com grau e tipo de exposição aos reservatórios, fatores ambientais, práticas agrícolas, e número de sorovares utilizados no exame.

Em relação aos caninos, dos 43 positivos, os sorogrupos que apresentaram maior soropositividade foram Canicola, com 19 $(44,19 \%)$, Pomona com $13(30,23 \%)$ e Icterohaemorrhagiae com $12(27,91 \%)$ animais reagentes. Em Botucatu (São Paulo), Coiro et al. (2011) encontraram maior reatividade aos sorogrupos Canicola, Pyrogenes, Sejroe e Djasiman. Os sorogrupos mais comumente associados à leptospirose canina clássica são Canicola e Icterohaemorrhagiae, e os roedores são tidos como reservatórios do último, o que 
pode indicar um contato dos cães amostrados com esse animal (Oliveira et al, 2013).

Dentre os 17 caninos selvagens positivos, os sorogrupos que mais apresentaram soropositividade ao teste SAM foram: Hebdomadis com oito $(47,05 \%)$ animais positivos e o Sejroe com seis $(35,29 \%)$ animais reagentes ao sorovar Hardjo e dois $(11,76 \%)$ ao sorovar Wolffi. Os bovinos são hospedeiros naturais do sorogrupo Sejroe (Figueiredo et al., 2009), o que justifica a presença desse sorogrupo nos caninos selvagens, já que estes animais usam áreas pecuárias como território, estando expostos ao contato com os bovinos (Rodrigues et al., 2015). Em estudos de Rodrigues et al. (2015) em canídeos de vida livre do cerrado brasileiro, os sorogrupos mais reagentes foram Icteroaemorrhagiae e Sejroe; os autores citam que o sorogrupo Icterohaemorrhagiae tem como um de seus principais reservatórios os roedores, que são potencialmente parte da cadeia alimentar destes animais.

Dos 106 caprinos testados, apenas um $(0,94 \%)$ animal foi reagente com titulação para o sorogrupo Hebdomadis. Diferente do presente estudo, Santos (2007) ao avaliar caprinos também em Uberlândia, MG, verificou a presença dos sorogrupos Autumnalis, Tarassovi, Pyrogenese e Icterohaemorrhagiae. O sorogrupo Hebdomadis foi relacionado como de maior prevalência em bovinos por Hashimoto et al. (2007) o que sugere que o animal reagente esteve em contato com bovinos.

Dos 17 equinos positivos, $11 \quad(64,70 \%)$ animais soroconverteram ao sorogrupo Sejroe sorovar Hardjo e cinco $(29,41 \%)$ o sorogrupo Canicola, sendo estes os sorogrupos que mais soroconverteram. Em oposição ao presente estudo, Lastra et al. (2013) analisaram equinos em Porto Alegre, RS, encontraram o sorogrupo Icterohaemorrhagiae como mais prevalente nos animais estudados. Souza et al (2016) também encontraram maior soropositividade para $\mathrm{o}$ sorogrupo Icterohaemorrhagiae em equinos de Uberlândia, MG. O sorogrupo Canicola é mais associados à leptospirose canina (Oliveira et al, 2013), e o sorogrupo Sejroe tem bovinos como hospedeiros naturais (Figueiredo et al., 2009), o que sugere que os equinos do estudo podem ter tido contato com estes animais.
Dos 35 felinos testados não houve animais reagentes em nenhuma titulação para nenhum sorogrupo testado. $\mathrm{O}$ mesmo aconteceu no estudo de Mittestainer et al. (2015) ao avaliar 155 felinos em Botucatu, São Paulo. Segundo os autores, o fato desses animais raramente serem reagentes para leptospira pode se dar ao fato de eles serem mais resistentes à infecção, embora existam relatos de gatos com leptospirúria após inoculação experimental. Sendo assim não se deve descartar a importância desses animais na disseminação da doença.

Dentre os 40 ovinos testados, um $(2,5 \%)$ animal foi positivo para o sorogrupo Tarassovi. Em contraponto ao ocorrido neste estudo, no município de Monte Negro, Rondônia, Aguiar et al. (2010) avaliaram amostras sanguíneas de ovinos e encontraram os sorogrupos mais frequentes Semaranga, Autumnalis, Australis, Sejroe, Icterohaemorrhagiae, Ballum e Hebdomadis. O sorogrupo Tarassovi já foi relacionado em ovinos no Rio Grande do Sul, em estudo de Herrmann et al. (2004).

Dos 17 roedores positivos, as maiores soropositividades foram para os sorogrupos Icterohaemorrhagiae com $12 \quad(70,58 \%)$, Pyrogenes com $10(58,82 \%)$ e Canicola com 8 $(47,06 \%)$ animais reagentes. Oliveira et al. (2014) avaliaram roedores comuns ao estado do Rio Grande do Sul, Brasil, e encontraram como sorogrupos mais aparentes sorogrupo Sejroe e o sorogrupo Pomona, mas também foi encontrado o sorogrupos Icterohaemorrhagiae, Grippotyphosa e Pyrogenes. A alta prevalência do sorogrupo Canicola levanta a hipótese do contato destes roedores com canídeos, já que este é mais associados à leptospirose canina (Oliveira et al, 2013). Roedores são reservatórios do sorogrupo Icterohaemorrhagiae (Oliveira et al., 2013; Rodrigues et al., 2015), o que justifica a alta prevalência no presente estudo.

Os 3 suínos positivos apresentaram titulação para três sorogrupos: Icterohaemorrhagiae, Pomona e Australis. Osava et al. (2010) também no município de Uberlândia, encontrou o sorogrupo Icterohaemorrhagiae como mais reagente, seguido do sorogrupo Sejroe. Esses dados concordam com o aparecimento do sorogrupo Icterohaemorrhagiae em suínos em Uberlândia. 


\section{Conclusão}

A leptospirose está presente na região estudada, em diferentes espécies animais, onde a bovina se mostrou a mais reagente, a caprina, a que menos reagiu, e a felina, a não reagente. $\mathrm{O}$ sorogrupo que mais reagiu foi o Sejroe.

\section{Referências}

Acha, P. N.; Szyfres, B. Zoonosis y enfermedades transmisibles comunes al hombre y a los animales. Washington: Organización Panamericana de laSalud, (Publicación Cientifica n. 503), 3 ed, Whashington, 2003. http://iris.paho.org/xmlui/bitstream/handle/123456789/71 1/9275119936.pdf

Adler, B. (Ed.). Leptospira and Leptospirosis: current topics in microbiology and immunology. Clayton, Australia: Springer, 2015. 387p.

Aguiar, D.; Cavalcante, M. G. T.; Vasconcellos, S. A.; Souza, G. O., Labruna, M. B., Camargo, L. M. A., Gennari, S. M. Anticorpos antiLeptospiras pp. em ovinos do município de Monte Negro, estado de Rondônia. Arquivo Instituto Biológico. v.77, n.3, p.529-532, 2010.

https://bdpi.usp.br/bitstream/handle/BDPI/1974/art_VAS CONCELLOS_Anticorpos_Anti_Leptospira_spp_em_ovi nos 2010.pdf?sequence $=1$

BRASIL. Ministério da Saúde. Fundação Nacional de Saúde. Centro Nacional de Epidemiologia. Coordenação de Controle de Zoonoses e Animais Peçonhentos. Manual de leptospirose. $2^{\mathrm{a}}$ edição, Brasília: Fundação Nacional de Saúde. 8 p. 1995.

BRASIL. VIGILÂNCIA EM SAÚDE: Zoonoses. Brasília - DF: Ministério da Saúde. n. 22 (Série B. Textos Básicos de Saúde Cadernos de Atenção Básica). 2009.

Bharti, A. R.; Nally, J. E.; Ricaldi, J. N.; Matthias, M. A.; Diaz, M. M.; Lovett, M. A.; Levett, P. N.; Gilman, R. H.; Willig, M. R.; Gotuzzo, E.; Vinetz, J. M. Leptospirosis: a zoonotic disease of global importance. The Lancet Infectious Disease, v.3, n.12, p.757771, 2003. http://dx.doi.org/10.1016/S14733099(03)00830-2

Castro, J. R. de. Aspectos epidemiológicos e imunológicos da leptospirose canina no município de Uberlândia, MG. 2010. 99 f.
Dissertação (Mestrado em Ciências Veterinária) - Curso de Medicina Veterinária, Programa de Pós Graduação em Ciências Veterinárias, Universidade Federal de Uberlândia, 2010. https://repositorio.ufu.br/bitstream/123456789/12970/1/D iss\%20Jacuqeline.pdf

Coiro, C. J.; Langoni, H.; Silva, R. C.; Ullmann, L. S. Fatores de risco para leptospirose, leishmaniose, neosporose e toxoplasmose em cães domiciliados e peridomiciliados em Botucatu-SP. Veterinária e Zootecnia. v.18, n.3, p.393-407, 2011. https://repositorio.unesp.br/bitstream/handle/11449/14089 2/ISSN0102-5716-2011-18-03-393407.pdf?sequence $=1$ \&isAllowed $=\mathrm{y}$

Eckstein, C.; Lopes, L. B.; Moustacas, V. S.; Rodrigues, R. O.; Castro, B. G.; Santos, R. L. Diagnosis of Leptospira spp. infection in sheepflocks in the State of Mato Grosso, Brazil. Acta Scientiae Veterinariae, v.45, p.15, 2017. https://pdfs.semanticscholar.org/e1fb/bd37666197b0add5 13a917ded57a60b5b69c.pdf

Favero, A. C. M.; Pinheiro, S. R.; Vasconcellos, S. A. et al. Sorovares de leptospiras predominantes em exames sorológicos de bubalinos, ovinos, caprinos, equinos, suínos e cães de diversos estados brasileiros. Ciência Rural, v.32, n.4, p.613-619, 2002. http://dx.doi.org/10.1590/S0103-84782002000400011

Figueiredo, A. de O.; Pellegrin, A. O.; Gonçalves, V. S. P.; Freitas, E. B.; Monteiro, L. A. R. C.; Oliveira, J. M.; Osório, A. R. Prevalência e fatores de risco para leptospisore em bovinos do Mato Grosso do sul. Pesquisa Veterinária Brasileira, v.29, n.5, p.375-381, $2009 . \quad$ http://dx.doi.org/10.1590/S0100736X2009000500003

Hashimoto, V. Y.; Gonçalves, D. D.; Silva, F. G.; Oliveira, R. C.; Alves, L. A.; Reichmann, P.; Muller, E. E.; Freitas, J. C. de. Occurrence of antibodies against Leptospira spp. in horses of the urban area of Londrina, Paraná, Brazil. Revista Instituto Medicina Tropical São Paulo, v.49, n.5, p.327-330, 2007. http://dx.doi.org/10.1590/S0036-46652007000500010

Herrmann, G. P.; Lage, A. P.; Moreira, E. C.; Haddad, J. P. A.; Resende, J. R.; Rodrigues, R. O.; Leite, R. C. Seroprevalence of agglutinins anti-Leptospira spp. in sheep from the Southeast and Southwest Mesoregions of the 
state of Rio Grande do Sul, Brazil. Ciência Rural, v.34, n.2, p.443-448, 2004. http://dx.doi.org/10.1590/S0103-84782004000200017

IBM SPSS Statistics: Health Science. IBM Corporation, 2013.

Langoni, H.; Del Fava, C.; Cabral, K. G.; Silva, A. V. da; CHagas, S. A. P. Aglutininas antileptospíricas em búfalos do Vale do Ribeira, estado de São Paulo. Ciência Rural, v.29, n.2, p.305-307, 1999. http://dx.doi.org/10.1590/S0103-84781999000200019

Lastra, C. S.; Oliveira, S. T.; Merini, L. P.; Dasso, M. G.; Pedralli, V.; González, F. H. D. Pesquisa de aglutininas anti-Leptospira em soros de equinos de tração em Porto Alegre, Brasil. Revista Brasileira de Ciências Veterinárias, v.20, n.1, p.23-25, 2013. http://dx.doi.org/10.4322/rbcv.2014.051

Levett, P. N. Leptospirosis. Clinical Microbiology Veterinary, v.14, p.296-326, 2001.

Martins, G.; Lilenbaum, W. The panorama of animal leptospirosis in Rio de Janeiro, Brazil, regarding the seroepidemiology of the infection in tropical regions. BMC Veterinary Research, v.9, n.1, p.237-252, 2013. http://dx.doi.org/10.1186/1746-6148-9-237

Mittestainer, J. C.; Melchert, A.; Ribeiro, J. F. A.; Sartori, R. S.; Joaquim, S. F.; Bresciani, K., Langoni, H. Estudo soroepidemiológico da infecção por Leptospiraspp. em gatos. Veterinária e Zootecnia. v.22, n.3, p.465-470, $2015 . \quad$ https://docplayer.com.br/67370249-Estudosoroepidemiologico-da-infeccao-por-leptospira-spp-emgatos.html

ORGANIZAÇÃO MUNDIAL DE SAÚDE ANIMAL (OIE). Terrestrial Manual. Leptospirosis. 2014. chap. 2.1.9. < http://www.oie.int/eng/normes/mmanual/a 00043.htm $>$. Acesso em: 20 de abril de 2016.

Oliveira, F. C. S. Fatores de risco para a leptospirose em fêmeas bovinas em idade reprodutiva no Estado da Bahia, Nordeste do Brasil. Pesquisa Veterinária Brasil, v.30, n.5, p.398-402, 2010. http://dx.doi.org/10.1590/S0100736X2010000500004

Oliveira, G. C.; SIlva, D. B.; Pinheiro, V. L. C.; Lagoni, H. Pesquisa de anticorpos anti- leptospíricos em bubalinos. ARS Veterinária, v.29, n.4, p.33, 2013 . http://dx.doi.org/10.15361/2175-0106.2013v29n4p33

Oliveira, S. T.; Stein, G. G.; santos, A. P.; biondo; A. W.; Messick, J. B.; Gonzalez, F. H. D. Serological evidence of Leptospira spp. in thelamituco-tucorodents (Ctenomyslami). Archives of Veterinary Science. v.19, n.3, p.69-72,

2014.

http://dx.doi.org/10.5380/avs.v19i3.35031

Oliveira, S. V.; Arsky, M. de L. N. S.; Caldas, E. P. Reservatórios animais da leptospirose: Uma revisão bibliográfica. Saúde (Santa Maria), v.39, n.1, p.9-20, 2013. http://dx.doi.org/10.5902/223658345094

Osava, C. F.; Salaberry, S. R. S.; Nascimento, C. C. N.; Lima-Ribeiro, A. M. C.; Moreira, R. Q.; Castro, J. R.; Rigo, V. H. B. Ocorrência de anticorpos anti-Leptospira spp. em diferentes sistemas de criação de suínos. Bioscience Journal, v.26, n.2, p.202-207, 2010. http://www.seer.ufu.br/index.php/biosciencejournal/articl e/view/7062/4680

Rodrigues, T. C. S.; Santos, A. L. Q.; Lima, A. M. C.; LemOS, F. G.; Azevedo, F. C.; Arrais, R. C.; Gomes, D. O.; Tavares, T. C. F. Occurrence of antibodies against Leptospira spp. in free-ranging wild canids from the brazilian savanna. Pesquisa Veterinária Brasileira, v.35, n.8, p.734-740, 2015. http://dx.doi.org/10.1590/S0100-736X2015000800005

Santos, J. P. Soroprevalência e aspectos epidemiológicos da leptospirose caprina no município de Uberlândia, MG. 2007. Dissertação (Mestrado em Ciências Veterinárias) - Curso de Medicina Veterinária, Programa de Pós Graduação em Ciências Veterinárias, Universidade Federal de Uberlândia, 2007. http://repositorio.ufu.br/bitstream/123456789/13171/1/jan dra.pdf

Souza, M. A.; Castro, J. R.; Moreira, R. Q.; Bombonato, N. G.; Soares, O. M.; Lima, A. M. C. Anti-Leptospira spp. antibodies in several animal species on the same farm. Bioscience Journal, v.32, n.1, p.202-207, 2016. http://www.seer.ufu.br/index.php/biosciencejournal/articl e/view/26605/17785 